\title{
Die Mauer, die man immer wieder überwinden konnte. Aus den Erfahrungen und den Erinnerun- gen eines polnischen Germanisten
}

\begin{abstract}
Podstawowa teza prezentowanego tu tekstu brzmi, że germanistyce polskiej czasów ostatnich dwu dekad PRL-u przysługiwały znaczne ,przestrzenie wolności ‘. Formułując tak wyostrzoną tezę, autor opiera się na własnych doświadczeniach i obserwacjach. Gromadził je jako badacz, eseista i recenzent.

Die Grundthese des nachfolgenden Textes besagt, dass der polnischen Germanistik in den letzten zwei Dekaden des, realsozialistischen' Systems im Grunde genommen (die freilich vorhandenen Einschränkungen und immer wieder vorkommenden Ausnahmen sollen keineswegs geleugnet werden) große Freiheitsräume von diesem System zugestanden wurden. Diese seine Grundthese exemplifiziert der Verfasser an Erfahrungen, die ihm selbst zuteil wurden. Sie sind insoweit aussagekräftig, als der Autor schon seit 1968 in der polnischen Germanistik als Forscher, Essayist und Rezensent tätig war.

In the present paper reference is made to the projected, for the early 2009 , periodical Convivium. On the basis of my long term experience I describe various relations, which in the times of Peoples Republic of Poland existed between the Polish centres of German studies and the corresponding centres existing in the West.
\end{abstract}

Der zahllosen menschlichen Tragödien oder einfach Erschwernisse, die die seit 1945 bis 1989 andauernde politische, soziale, kulturelle Teilung Europas, die die quer durch den Kontinent gezogene Mauer aus Aggressivität, Feindseligkeit und Hass bewirkt hat, bin ich mir mit aller notwendigen Klarheit durchaus bewusst. Diese an sich eigentlich selbstverständliche Feststellung sei vorneweg formuliert, damit mich niemand der Unkenntnis der Sachlage oder gar der Verharmlosung der kommunistischen Regimes zeiht. Trotz 
alledem: In dem von mir persönlich erlebten und erfahrenen Zeitraum existierte diese Mauer so gut wie überhaupt nicht. Wenn man also den Begriff der Mauer für die Geschichte unserer Fachdisziplin überhaupt aufrecht erhalten möchte, so müsste man sofort mit Nachdruck anmerken, dass diese Mauer an vielen Stellen durchlöchert war und dass man sie insoweit fast völlig frei in beiden Richtungen passieren konnte.

Um nun also endlich das angesagte Thema konkret anzugehen: Im Jahre 1963 habe ich das Germanistikstudium an der Universität Wroclaw aufgenommen und es 1968 an der Universität Leipzig abgeschlossen. Seit 1968 bis 1989 (auf diesen Zeitraum sollen meine Ausführungen begrenzt sein) war ich als Forscher und akademischer Lehrer in der polnischen Germanistik tätig: zunächst bis 1981 in Wroclaw und danach in Rzeszów. Im Jahre 1991 bin ich in den Rang des Professors für die Geschichte der neueren und auch der neuesten deutschsprachigen Literatur aufgestiegen. Nun soll die erste Mitteilung kommen, die wohl all diejenigen, die sich von dem ,realsozialistischen ' Polen ein Bild Grau in Grau malen, völlig überraschen wird. In der genannten Zeit durfte ich ohne jegliche Kontrolle und ohne jegliche Einschränkung jedes im Westen erschienene Werk, sei es aus dem Bereich der Primär-, sei es aus dem Bereich der Sekundärliteratur, lesen und studieren. Es erging mir noch besser, viel besser. Wenn mich ein in den deutschsprachigen Ländern des Westens publiziertes Buch interessierte oder wenn ich es gar als nützlich für meine Tätigkeit als Forscher, Essayist oder Rezensent erachtete, konnte ich es einfach geschenkweise, also ohne den geringsten Kostenaufwand meinerseits, von einer der vielen bundesdeutschen Kulturstiftungen erhalten. Die notwendige Voraussetzung dafür war freilich, dass man über die deutschsprachige Literatur schrieb und publizierte. Diese Bedingung erfüllte ich. Ich darf mit einigem Stolz sagen, dass ich ab etwa 1970 regelmäßig größere Artikel über deutsche Schriftsteller und deutsche Literaturwerke veröffentlichte, dank der Hochherzigkeit vieler Redaktionen veröffentlichen durfte. Schon im Jahre 1971 wurde ich von dem Chefredakteur der Kulturzeitschrift Odra (Wrocław), die damals in einer Auflage von 10.000 Exemplaren erschien, dazu , auserkoren', eine dreiteilige Arbeit über die faschistische Literatur präsentieren zu dürfen. Eine gewisse Leistung war damit erbracht. Weitere Texte kamen bald hinzu. So konnte ich daran denken, mit meinen Bücherwünschen bei den dafür zuständigen bundesdeutschen Kulturstiftungen vorstellig zu werden. Und für die Dauer von beinahe zwanzig Jahren konnte ich auch auf ihre Hilfsbereitschaft stets rechnen. Wie war das möglich? Die Antwort ist einfach. Die Bundesrepublik Deutschland verfolgte 
eine kluge Politik, wozu gehörte, dass sie diejenigen förderte, die in ihren Herkunftsländern die Anliegen der deutschen Literatur verfochten.

Allerdings: Im Hinblick auf den oben erörterten Aspekt ist die Situation der Germanisten nach dem Ende des polnischen Kommunismus eigentlich schlechter geworden. Denn als Polen seine demokratische Freiheit wiedergewann und sich ins demokratische Europa wiedereingliederte, hörten bundesdeutsche Stiftungen, ob staatlich oder nichtstaatlich, überhaupt auf, germanistische Literaturwissenschaftler aus Polen kostenlos mit Büchern zu versorgen! Die unmittelbare, unvermeidliche und dabei höchst schmerzliche Folge war, dass ich, dass alle polnischen Germanisten von diesem Augenblick an die in den deutschsprachigen Ländern publizierten Bücher selbst bezahlen mussten! Man soll mich nicht missverstehen: Ich beklage es nicht im geringsten... Es ging durchaus mit rechten Dingen zu. Mit nüchternem Verständnis stelle ich die nackte Tatsache fest: Unter radikal veränderten politischen und kulturpolitischen Verhältnissen sahen sich die polnischen Germanisten dazu gezwungen, die von ihnen für ihre Forschungsarbeit benötigten Bücher aus der Bundesrepublik Deutschland oder aber aus Österreich vom eigenen Verdienst zu finanzieren. Und weil das Einkommensgefälle zwischen Polen und z.B. der Bundesrepublik Deutschland unverändert groß war, so wurden die westlichen Publikationen für sie in vielen Fällen unerschwinglich. Meine Pointe lautet: eine ,Mauer', eine nur schwer übersteigbare Mauer, ist erst in dieser Zeit entstanden.

Zum Situationsbild der polnischen Germanistik in den 70er und 80er Jahren gehört freilich auch die betrübliche Tatsache, dass selbst angesehenste Universitätsbibliotheken so gut wie gar nicht über sog. ,harte Währungen“ verfügten. Wenn es einmal im Jahre ,hochging“, konnte eine Universitätsbibliothek in einer großen Stadt (Warszawa, Wrocław, Kraków) bestenfalls einige Dutzend Bücher aus dem deutschsprachigen westlichen Ausland bestellen. Zum Glück galt auch für die offiziellen akademischen Institutionen (hier sind die Bibliotheken natürlich mitgemeint) das Verfahrensprinzip, von dem, wie gerade dargelegt, ebenfalls ich als Einzelperson wieder und wieder im Laufe der vielen Jahre profitieren durfte. Auch germanistische Institute wurden von bundesdeutschen sowie österreichischen Kulturstiftungen freigebig mit Büchern beschenkt. Ich hatte die allerbeste Möglichkeit, dies an der kleinen, erst kürzlich gegründeten Germanistik in Rzeszów zu beobachten. Ab 1982 stand ich ihr nämlich als Direktor vor. Aufgrund amtlicher Befugnisse konnte ich also bis auf das letzte Tüpfelchen, auf die letzte Stelle hinter dem Komma erfahren, was alles an Buchspenden nach Rzeszów aus 
dem ,Westen“ kam. Der Wert der alljährlichen Geschenkgaben belief sich auf mehrere Tausend Euro. Nicht anders als im Falle von einzelnen germanistischen Forschern ist nach 1989 diese Spendenquelle auch für alle universitären Anstalten versiegt.

Um aber in die Zeit vor der ,Wende“ zurückzugehen: Nicht nur dank der Buchgeschenke war ich in der Lage zu verfolgen, was sich in der neuesten Literatur der deutschsprachigen Länder oder aber in der germanistischen Literaturwissenschaft tat. Die mir in dieser Hinsicht zu Gebote stehenden Möglichkeiten wurden noch zusätzlich dadurch gesteigert, dass ich als Stipendiat des DAAD oder der Friedrich-Ebert-Stiftung in den 80er Jahren mehrmals, eigentlich jedes zweite Jahr, in die Bundesrepublik reisen und, von den Geldmitteln der bundesdeutschen Kulturinstitutionen profitierend, diverse germanistische Institute aufsuchen und in ihren Bibliotheksbeständen stöbern konnte. Von bundesdeutschen oder österreichischen Stipendien konnten die meisten meiner Kolleginnen und Kollegen Gebrauch machen!

Mit den sog. „Westreisen“ sind manche schlimmen Gerüchte verbunden. Sie sind nicht dem Nichts entsprungen. Tatsächlich wurde damals die Passpolitik oft als Erpressungsmittel benutzt. Die diesbezüglichen Praktiken spielten sich freilich im Geheimen ab. Inwieweit sie auch die Germanisten betrafen, kann ich also nicht beurteilen. Mir sind jedenfalls Kontakte mit dieser zwielichtigen Sphäre erspart geblieben. Bei der Aushändigung des zu einer Reise in den Westen berechtigenden Passes hat mir kein einziges Mal die Passbehörde auch nur geringfügigste Schwierigkeiten gemacht. Ich hatte auch das Glück, dass diese Instanzen kein einziges Mal versucht haben, mit mir ins Gespräch zu kommen, um mich im Zusammenhang mit dem geplanten Aufenthalt in dem ,Feindesland“ Westdeutschland für eine wie auch immer geartete Mitarbeit zu gewinnen. Und über den Verlauf des jeweiligen Studienaufenthaltes musste ich keine Berichte, und sei es an eine akademische Instanz, abliefern. So hatte ich das an Sicherheit grenzende Gefühl, dass ich mich im Westen vollkommen frei tummeln und mir als Forscher alles herausnehmen durfte.

Eigentlich bin ich am Ende meiner Erinnerungsarbeit angelangt. Dem Thema „germanistische Freiheitsräume im Ostblock“ möchte ich noch eine weitere, eigentlich unwahrscheinlich anmutende Geschichte nachtragen. 1963 habe ich das Germanistikstudium an dem Germanistischen Institut in Wrocław aufgenommen. Im Jahre 1965 haben mich meine Lehrmeister in Wrocław für würdig befunden, ab Herbst mein Studium schon im Ausland fortzusetzen. Selbstverständlich kam von allen deutschsprachigen Ländern nur die DDR in Frage. So ist es dann auch gekommen. Am 1. Oktober traf ich in Leipzig ein 
und meldete mich auf der Stelle im dortigen Germanistischen Institut. Bis zum Studienabschluss, den ich 1968 geschafft habe, sollte es meine germanistische Heimat bleiben. Hier fängt die eigentliche, allen gängigen Vorstellungen über den damaligen Studienbetrieb in dem damals zu Recht als eine schlimme Diktatur verschrieenen Ulbricht-Staat widersprechende Story an. Denn wer immer dort Germanistik zu studieren beabsichtigte, musste sich eigentlich darauf gefasst machen, dass man ihn sofort in die ideologische Zange nehmen, seine Lektüren und auch seine Ansichten überwachen und überhaupt im didaktischen Prozess nur das anbieten werde, was mit den Prinzipien des ,heiligen“ Marxismus und Leninismus sich vereinbaren lasse. Und generell galt dies fast ohne Ausnahmen. Mit der polnischen Studentengruppe, der ich angehörte, ist indes die Sache völlig anders verlaufen. In den drei Jahren, in denen man mich in Leipzig Geschichte der deutschen Literatur lehrte, wurde ich in keinem einzigen Seminar, in keiner einzigen Vorlesung der ideologischen Pression ausgesetzt. Mehr noch: Von den im politischen Sinne ,korrekten', also marxistisch eingestellten Autoren wurde in ihnen überhaupt nicht gesprochen. Ich (und andere Angehörige der polnischen Studentengruppe) bekamen dagegen die Möglichkeit, uns mit Werken von ,unmarxistischen', ,spätbürgerlichen', gar ,dekadenten' Autoren vertraut zu machen. ${ }^{1}$ Um es wenigstens mit einem Beispiel zu belegen. Das erste Literaturseminar, das uns angeboten wurde, rückte Schriftsteller wie - man möchte es gar nicht glauben - Rainer Maria Rilke und Gottfried Benn in den Vordergrund. Und wir wurden in ihr Schaffen mit behutsamer Sachlichkeit, ohne jegliches ideologisches Raster eingeführt.

Ich fahre in meinen Erinnerungen an die Leipziger Studienzeit fort. Meine Diplomarbeit betreute der zu den führenden Literaturtheoretikern der DDR gezählte Prof. Claus Träger. Er war ein orthodoxer Marxist und sein besonderes Erkennungszeichen war eine scharfe Feder, die er als ein oft tödlich wirkendes Instrument einsetzte, mit dem Autoren angegriffen wurden, wenn sie sich irgendeines Vergehens gegen den dogmatisch verstandenen Marxismus schuldig gemacht haben. Mich aber hat der inquisitorische Professor auf eine Art und Weise behandelt, dass ich ihn in dieser Hinsicht heute noch nicht genug rühmen kann. Er ließ es ohne jeglichen Einwand zu, dass ich meine Diplomarbeit Autoren wie Franz Kafka, William Faulkner und Albert Camus widmete. Jedermann, der irgendetwas über die Literatur des ver-

1 Ich gebrauche die Terminologie jener tatsächlich zu einseitigen Be- und Abwertungen neigenden Epoche. Was mit den angeführten Begriffen gemeint war, weiß heutzutage so gut wie keiner mehr. 
gangenen Jahrhunderts weiß, sieht sofort, dass es sich um Schriftsteller handelte, die man für den Marxismus nicht im Entferntesten in Anspruch nehmen könnte. In der DDR waren sie damals so gut wie Unpersonen. Ihre Werke wurden so gut wie nicht publiziert, die Literaturhistoriker befassten sich mit ihnen nicht, in der Kulturpresse wurden sie nur selten erwähnt. Und wenn dies geschah, sprach man über sie äußerst kritisch. Und wie ging ich vor? Über ,meine" Autoren schrieb ich mit Hochachtung. Es fiel mir gar nicht ein, bei der Analyse ihrer Werke irgendwelche marxistischen Bewertungskriterien mit zu bedenken. Eigentlich hätte ich mit einer strengen Rüge rechnen müssen. Aber Prof. Claus Träger reagierte auf meine Arbeit sehr wohlwollend, eigentlich enthusiastisch. Ich bekam eine Note, die es formell gar nicht gegeben hat: „exzellent“. Was hat sich da eigentlich abgespielt? Ich nehme an, dass dieser menschlich übrigens sehr eindrucksvolle Mann mich, einen jungen Germanisten aus Polen, einfach gemocht hat. Vor allem wohl deswegen, weil er sofort bemerken konnte, dass ich wirklich ein passionierter Liebhaber des deutsch geschriebenen Wortes war. Aber gewiss zeigt das damals zwischen ihm und mir Vorgefallene, dass dieser germanistische Dogmatiker und literaturkritische Scharfrichter sich auch großherzig zu verhalten verstand. Ein Sonderfall? Gewiss. Aber auch Sonderfälle sind Fälle und haben ihren Erkenntniswert. 